\title{
Construyendo puentes interdisciplinarios: la Red de Ciencias Aplicadas a la Investigación y Conservación del Patrimonio Cultural (Red-CAICPC), México
}

Building Interdisciplinary Bridges: the Red de Ciencias Aplicadas a la Investigación y Conservación del Patrimonio Cultural (Red-CAICPC, Applied Sciences Network for Cultural Heritage Research and Conservation), Mexico

\section{Luis Barba Pingarrón}

Instituto de Investigaciones Antropológicas (IIA),

Universidad Nacional Autónoma de México (UNAM), México

barba@unam.mx

\section{Isabel Medina-González}

Escuela Nacional de Conservación, Restauración y Museografía (ENCRYM), Instituto Nacional de Antropología e Historia (INAH), México

isabel_medina_g@encrym.edu.mx

\section{Resumen}

El principal propósito de la Red de Ciencias Aplicadas a la Investigación y la Conservación del Patrimonio Cultural (Red-CAICPC), iniciativa financiada por el Consejo Nacional de Ciencia y Tecnología (Conacyt), México, es fomentar la cooperación e intercambio entre expertos, laboratorios e institutos dedicados a las ciencias aplicadas para que construyan de manera colectiva e interdisciplinaria investigaciones, procedimientos y metodologías en materia de estudio, conservación, gestión y difusión del patrimonio cultural. Esta SEMBLANZA informa sobre los antecedentes, metas estrategias, organización y participantes de la red, así como sus logros, todo ello con el fin de establecer sus horizontes.

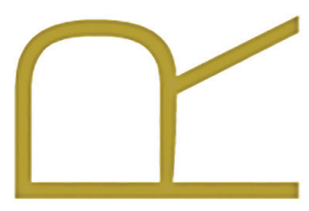

\section{Palabras claves}

ciencia; patrimonio cultural; red; investigación; conservación; México

\section{Abstract}

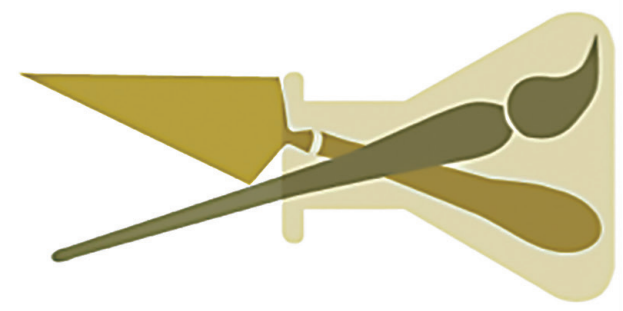

The principal aim of the Red de Ciencias Aplicadas a la Investigación y la Conservación del Patrimonio Cultural (Red-CAICPC, Applied Sciences Network for Cultural Heritage Research and Conservation), an initiative sponsored by Consejo Nacional de Ciencia y Tecnología (Conacyt, National Council for Science and Technology), Mexico, is to promote cooperation and exchange amongst 
applied sciences experts, laboratories and organisations in order to facilitate collective and interdisciplinary research, procedures and methodologies for the study, conservation, management and dissemination of cultural heritage. This OVERVIEW focuses on the Red-CAICPC's background, strategic objectives, organization, and participants; it also analyses the network's achievements to set up its future goals.

\section{Key words}

science; cultural heritage; network; research, conservation, Mexico

\section{Introducción}

El patrimonio cultural de México, de gran riqueza y diversidad, no sólo es un elemento esencial de investigación sobre nuestra historia: al derivar del reconocimiento y la valoración de nuestro pasado, también lo es de nuestra identidad, amén de un factor de desarrollo comunitario, económico y humano. Por ello, y en virtud de los desafíos que significan tanto su preservación en el presente como su transmisión al futuro, la gestión, el manejo y la conservación patrimonial han de acometerse desde una perspectiva estratégica, interdisciplinaria y de cooperación.

Si bien la conjunción de ciencia y patrimonio no es nueva en México: existe una amplia tradición de estudios de materialidad y tecnología en las disciplinas de la arqueometría y la restauración (cfr. Magaloni 1990; FiIloy 1992; Medina-González 1998; Barba 2000), lo notable de hoy en día es que también contamos con una vasta infraestructura científica y tecnológica de frontera a lo largo del país, cuyo empleo facilita el estudio de objetos y estructuras patrimoniales en proceso de investigación, diagnóstico, monitoreo y evaluación (Figura 1). Nuestra situación privilegiada no se limita a esto: poseemos, además, personal capacitado y experimentado en el desarrollo de estudios científicos patrimoniales (cfr. Barba 2013). Y aunque la investigación científica no acaba de ser una práctica generalizada en una gran cantidad de proyectos de indagación e intervención patrimonial, la relación entre arqueólogos, restauradores y científicos es prolija, de gran calidad y estratégica para la toma de decisiones, tal y como lo demuestra una profusa bibliografía (Cabrero y Ruvalcaba-Sil 2013; Chi Keb et al. 2013; Cucina 2013; Filloy Nadal et al. 2013; GarcíaHernández et al. 2013; Götz 2013; Götz y Stanton 2013; McGlinchey et al. 2013; Ortega y Tiesler 2013; Perea et al. 2013; Price et al. 2013; Ruvalcaba-Sil et al. 2013; Sánchez y Álvarez 2013; Tiesler et al. 2013a; Tiesler et al. 2013b). No obstante lo anterior, esta rica vinculación requiere ahora formalizarse para que alcance su verdadero potencial.

Ésta es, precisamente, una de las misiones de la Red de Ciencias Aplicadas a la Investigación y la Conservación del Patrimonio Cultural (Red-CAICPC) (Figura 2), iniciativa financiada por el Consejo Nacional de Ciencia y Tecno-

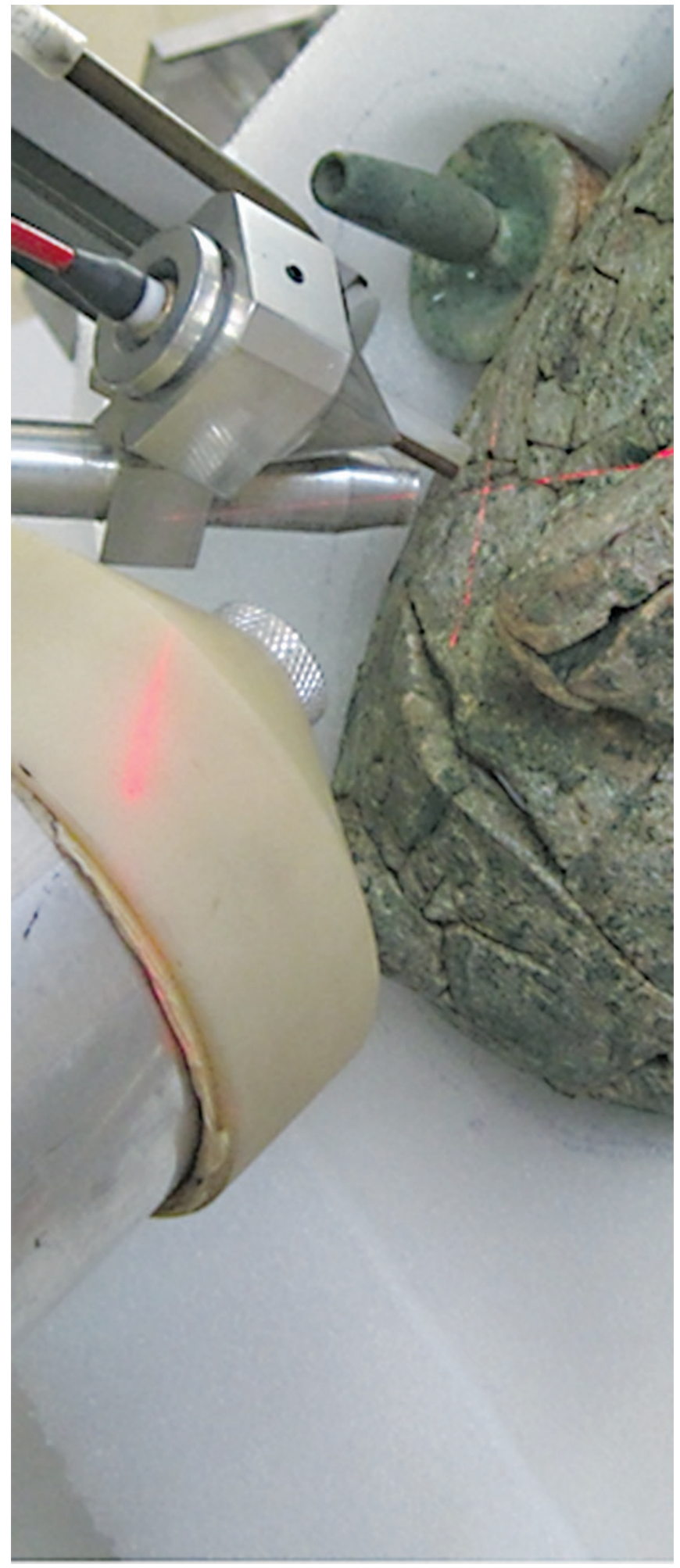

FIGURA 1. Estudio de la máscara funeraria de K'inich Janaab' Pakal, (Cortesía: INAH e IF-UNAM).

logía (Conacyt) que busca establecer, de manera colectiva e interdisciplinaria, procedimientos y metodologías de trabajo enfocados en el estudio del patrimonio cultural, primeramente, mediante la participación de personal altamente capacitado $y$, en segundo lugar, con el equipa- 
miento científico adecuado para asegurar que aquél y éste sean el sustento de las iniciativas de investigación, conservación, gestión y difusión en nuestro país (Barba 2013).

\section{Propósitos}

La Red-CAICPC tiene como propósito general:

Vincular especialistas en áreas de ciencias aplicadas al estudio del patrimonio para impulsar un esfuerzo interdisciplinario que conforme grupos de investigación en técnicas analíticas, de prospección y de datación, en ciencias biológicas y de la tierra, así como en historia del arte, arqueología y conservación, para favorecer un mayor conocimiento y un mejor diagnóstico de la materialidad que constituye al patrimonio cultural del país con fines de su investigación, conservación y difusión (Figura 2).

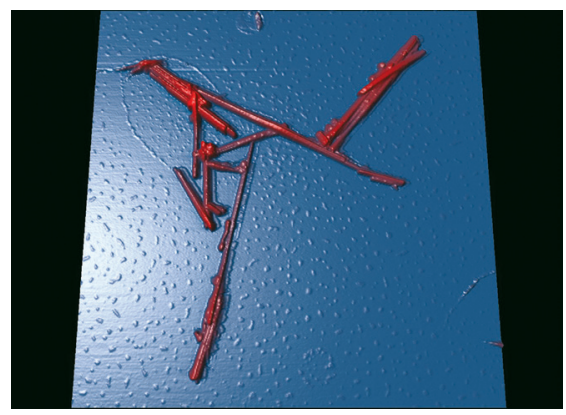

FIGURA 2. Estructura filiforme de paligorskita en muestra de pigmento azul maya, estudio por microscopia de sonda de barrido en modalidad no contacto (Cortesía: Laboratorio de microscopia electrónica y difracción de rayos $\mathrm{X}$, ININ).

Con base en ello, se han determinado los siguientes objetivos específicos:

- Unificar y desarrollar, con base en el intercambio de experiencias entre los laboratorios y grupos de investigación del país, las mejores prácticas en el uso de metodologías científicas para el conocimiento y la conservación del patrimonio cultural nacional

- $\quad$ Establecer protocolos comunes de medición e indagación; generar referentes certificados, e intercambiar conocimientos sobre estrategias experimentales y técnicas de estudio de dicho patrimonio

- Realizar a escala nacional intercambios de expertos y establecer colaboraciones con el fin de construir un saber patrimonial más integral, profundo y completo

- Formar recursos humanos especializados en el ámbito del estudio interdisciplinario del patrimonio cultural con enfoques regionales

- Integrar y compartir bases de información globales sobre las características de nuestros bienes culturales para su estudio y conservación

\section{Organización y participantes}

La Red-CAICPC se ha integrado con miembros de diversas regiones geográficas y especialidades: de esta manera se ha tratado de incorporar de manera equilibrada tanto las líneas de investigación existentes en el ámbito patrimonial como las instituciones clave en su estudio, conservación y difusión.

\section{COMITÉ ACADÉMICO DE LA RED-CAICPC}

Nombre

Institución

Dr. Luis Barba Pingarrón

(Responsable ante Conacyt)

Dr. Avto Gogichaishvili

Dr. José Luis Ruvalcaba-Sil

Dra. Clara Bargellini Cioni

Dr. Niklas Schulze

Dra. Blanca Maldonado Álvarez

Dra. Isabel Villaseñor Alonso

Dr. Javier Reyes Trujeque
Instituto de Investigaciones Antropológicas, Universidad Nacional Autónoma de México (IIA-UNAM)

Instituto de Geofísica, Universidad Nacional Autónoma de México (Geofísca-UNAM)

Instituto de Física, Universidad Nacional Autónoma de México (IF-UNAM)

Instituto de Investigaciones Estéticas, Universidad Nacional Autónoma de México (IIE-UNAM)

Universidad Autónoma de San Luis

Potosí (UASLP)

El Colegio de Michoacán, A. C.

(Colmich)

Coordinación Nacional de Conservación del Patrimonio Cultural, Instituto Nacional de Antropología e Historia (CNCPC-INAH)

Centro de Investigación en Corrosión, Universidad Autónoma de Campeche (Cicorr-UAC)

FIGURA 3. Integrantes del comité académico de la Red de Ciencias Aplicadas a la Investigación y la Conservación del Patrimonio Cultural (Red-CAICPC) (Cortesía: Red-CAICPC). 


\begin{tabular}{|c|c|}
\hline \multicolumn{2}{|c|}{$\begin{array}{c}\text { GRUPO DE TRABAJO DEL COMITÉ ACADÉMICO } \\
\text { DE LA RED-CAICPC }\end{array}$} \\
\hline Nombre & Institución \\
\hline Dra. Ana María Soler Arrechalde & $\begin{array}{l}\text { Instituto de Geofísica, Universi- } \\
\text { dad Nacional Autónoma de México } \\
\text { (Geofísica-UNAM) }\end{array}$ \\
\hline Mtra. Sandra Zetina Ocaña & $\begin{array}{l}\text { Instituto de Investigaciones Estéticas, } \\
\text { Universidad Nacional Autónoma de } \\
\text { México (IIE-UNAM) }\end{array}$ \\
\hline Dr. Manuel Espinosa Pesqueira & $\begin{array}{l}\text { Instituto Nacional de Investigaciones } \\
\text { Nucleares (ININ) }\end{array}$ \\
\hline Dr. Rodrigo Esparza López & $\begin{array}{l}\text { El Colegio de Michoacán, A. C. } \\
\text { (Colmich) }\end{array}$ \\
\hline Mtra. Jannen Contreras Vargas & $\begin{array}{l}\text { Escuela Nacional de Conservación, } \\
\text { Restauración y Museografía, Instituto } \\
\text { Nacional de Antropología e Historia } \\
\text { (ENCRYM-INAH) }\end{array}$ \\
\hline Dra. Laura Filloy Nadal & $\begin{array}{l}\text { Museo Nacional de Antropología, Insti- } \\
\text { tuto Nacional de Antropología e Historia } \\
\text { (MNA-INAH) }\end{array}$ \\
\hline
\end{tabular}

FIGURA 4. Integrantes del Grupo de Trabajo del Comité Académico de la Red de Ciencias Aplicadas a la Investigación y la Conservación del Patrimonio Cultural (Red-CAICPC) (Cortesía: Red(AICPC).

Para su funcionamiento, la red cuenta con un comité académico (Figura 3) y un grupo de trabajo asociado (Figura 4).

Actualmente participan en ella, además de diversos laboratorios, varios grupos de investigación e instituciones, cuyos responsables ante la Red-CAICPC se detallan en la Figura 5.

\section{Avances}

Los citados grupos de investigación han comenzado a disponer, gracias a los apoyos institucionales obtenidos de manera independiente, de infraestructura científica adecuada para enfrentar los retos planteados por el estudio y la conservación del patrimonio, con lo que se han ofrecido resultados notables tanto en las técnicas de prospección y datación, la caracterización de materiales con diversas técnicas y el estudio de biomateriales como en el diagnóstico y la conservación del patrimonio (CAICPC 2016). En la actualidad, la Red-CAICPC se ha sumado a estos esfuerzos con la finalidad de potenciar su crecimiento y consolidación, principalmente en dos sentidos: apoyando, por un lado, el desarrollo de grupos de investigación establecidos en el centro-norte, en el occidente y en el sureste del país, y, por el otro, la creación de grupos en regiones donde existe un notable patrimonio cultural, pero todavía escasa infraestructura y limitada investigación. Con base en una serie de reuniones nacionales, la más reciente en la Escuela Nacional de Conservación, Restauración y Museografía (ENCRYM) del Instituto Nacional de Antropología e Historia (INAH), en noviembre del 2015 (Figura 6), se han identificado, asimismo, agendas de investigación conjunta y potenciales ámbitos de intervención cientí- fica en la investigación patrimonial, particularmente en el campo metodológico. El buen efecto de estas iniciativas, por las que realmente se conoce el sentido de la interdisciplina en la escala del diálogo y la innovación de los saberes, se empieza a perfilar como uno de los más promisorios de la Red-CAICPC.

En breve, la suma de productos derivados del trabajo de investigación colaborativo de los grupos de la red ha hecho evidente su potencial para realizar investigación científica patrimonial de forma coordinada, interdisciplinaria y sumatoria entre diferentes laboratorios interinstitucionales, con un uso apropiado de las infraestructuras disponibles y la generación de transversalidad de saberes (cfr. Aguilar-Téllez et al. 2014; Alcántara 2014; Alonso et al. 2014; Arano et al. 2014; Castillo y Zimmermann 2014; Cucina y Ortega 2014; Cucina y Tiesler 2014; Cucina 2015; Espinosa-Morales et al. 2014; Espinosa y Arroyo 2014; Fernández et al 2014; García-Bucio et al. 2014; Götz 2014; Götz y Emery 2014; Götz et al. 2014; Herrera y Götz 2014; Jiménez et al. 2014; Manrique et al. 2014; Melgar y Ruvalcaba-Sil 2014; Muñoz et al. 2014; Ortega y Tiesler 2014; Quintana et al. 2014; Ramírez-Salomón et al. 2014; Riquelme et al. 2013, 2014a y 2014b; Sánchez y Álvarez 2014; Sierra et al. 2014; Solís y RuvalcabaSil 2014; Tiesler 2014; Tiesler et al. 2014; Tiesler y Cucina 2014; Velázquez-Castro 2014; Velázquez-Castro y Adje 2014; Velázquez-Castro y Melgar 2014; Villegas et al. 2014; Zetina et al. 2014; Zucchiatti et al. 2014). Debido a ello no sólo se ha obtenido un mayor conocimiento sobre la materialidad y la tecnología de nuestro patrimonio, fundamental para diagnosticar su deterioro y para proponer estrategias para su adecuada conservación, sino también se han establecido agendas de estudio con enfoques regionales.

Asimismo, el trabajo de la Red-CAICPC ha permitido, con el consecuente equilibrio de la asimetría en el desa- 


\section{LABORATORIOS, GRUPOS DE INVESTIGACIÓN E INSTITUCIONES PARTICIPANTES EN LA REd-CAICPC}

Laboratorio de Biodeterioro (LBD)

Centro Nacional de Conservación y Registro del Patrimonio Artístico Mueble-Instituto Nacional de Bellas Artes, (CENCROPAMINBA)

Mtra. Sandra Ramírez Muñoz
Laboratorio de Refinamiento de Estructuras Cristalinas (LAREC)

Instituto de Física-Universidad Nacional Autónoma de México (IF-UNAM)

Dr. Rubén Santamaría Ortiz
Laboratorio de Microscopía Electrónica (LME)

División de Arquitectura Arte y Diseño, Maestría en Restauración de Sitios y Monumentos-Universidad de Guanajuato (DAAD [MRSM]-UC)

Mtra. Dolores Elena Álvarez Gasca
Laboratorio de Prospección Arqueológica (LPA)

Instituto de Investigaciones Antropológicas-Universidad Nacional Autónoma de México (IIA-UNAM)

Dr. Luis Alberto Barba Pingarrón
Laboratorio de Cristalofísica y Materiales Naturales (LCMN)

Instituto de Física-Universidad Nacional Autónoma de México (IFUNAM)

Dr. Lauro Bucio Galindo
Cuerpo Académico de Química y Tecnología de Silicio (CAQTS)

Facultad de Química, Departamento de Química-Universidad de Guanajuato (FQ [DQ]-UG)

Dr. Jorge Cervantes Jáuregui
Laboratorio de Antropología Genética (LAG).

Instituto de Investigaciones Antropológicas-Universidad Nacional Autónoma de México (IIA-UNAM)

Dra. Blanca Zoila González Sobrino

Dra. Ana Julia Aguirre Samudio
Laboratorio Nacional de Espectrometría de Masas con Aceleradores (LEMA)

Instituto de Física-Universidad Nacional Autónoma de México (IF-UNAM)

Dra. Corina Solís Rosales

Dr. Efraín R. Chávez

Dra. María Rodríguez Ceja
Cuerpo Académico de Geología (CA-Geología)

División de Ingenierías-Universidad de Guanajuato (DI-UG)

Dr. Raúl Miranda Avilés

Dra. María Jesús Puy y Alquiza

Laboratorio Nacional de Caracterización de Propiedades Fisicoquímicas y Estructura Molecular (LACAPFEM)

Laboratorio Nacional de Ciencias para la Investigación del Patrimonio Cultural IIE-UNAM (LANCIC IIE-UNAM)

Instituto de Investigaciones Estéticas-Universidad Nacional Autónoma de México (IIE-UNAM)

Instituto de Química-Universidad Nacional Autónoma de México (IQ-UNAM)

M. en C. Baldomero Esquivel Rodríguez
Dirección de Apoyo a la Investigación y al Posgrado-Universidad de Guanajuato (DAIP-UG)

Dr. Jorge Albino López Jiménez
Mtra. Sandra Zetina Ocaña
Laboratorio Nacional de Ciencias para la Investigación y la Conservación del Patrimonio Cultural IF, UNAM (LANCIC IF, UNAM)

Instituto de Física, Laboratorio de Análisis no Destructivo para e Estudio In Situ del Arte, la Arqueología y la Historia -Universidad Nacional Autónoma de México (IF-ANDREAH-UNAM)

Instituto de Investigaciones Nucleares (ININ)

Dr. José Luis Ruvalcaba-Si

Laboratorio de Termoluminiscencia (L-TI)

Instituto de Geofísica-Universidad Nacional Autónoma de México (Geofísica-UNAM)

Dr. Peter Shaaf

M. en C. Ángel Ramírez Luna

M. en C. Ángel Ramírez Luna

Servicio Arqueomagnético Nacional (SAN)

Instituto de Geofísica, Unidad Michoacán-Universidad Nacional Autónoma de México (Geofísica [Unidad Michoacán]-UNAM)

Dr. Avto Gogichaishvili

Dr. Juan Morales

Laboratorio de Paleomagnetismo (LPm)

Instituto de Geofísica, Área de Arqueomagnetismo-Universidad Nacional Autónoma de México (Geofísica [AAm]-UNAM)

Dra. Ana M. Soler Arechalde
Laboratorio Nacional de Ciencias para la Investigación y la Conservación del Patrimonio Cultural ININ-UNAM (LANCIC ININ-UNAM) Instituto Nacional de Investigaciones Nucleares (ININ)

\section{(USAIQ).}

Unidad de Servicios de Análisis Instrumental y Químicos

División de Ciencias Naturales y Exactas, Departamento de Química-Universidad de Guanajuato (DCNE [DQ]-UG)

Dr. Manuel Eduardo Espinosa Pesqueira

Proyecto Técnicas de Manufactura de los Objetos de Concha del México Prehispánico (PTM-OCMP)

Museo del Templo Mayor-Instituto Nacional de Antropología e Historia (MTM-INAH)

Dr. Adrián Velázquez Castro

Taller de Arqueología Experimental y Análisis Tecnológico del Laboratorio de Microscopía Electrónica (TAEAT-LME)

Museo del Templo Mayor-Instituto Nacional de Antropología e Historia (MTM-INAH)

Dr. Emiliano Ricardo Melgar Tísoc

Laboratorio de Conservación, Diagnóstico y Caracterización Espectroscópica de Materiales (CODICE)

Coordinación Nacional de Conservación del Patrimonio CulturalInstituto Nacional de Antropología e Historia (CNCPC-INAH)

M. en C. Nora Pérez Castellanos

M. en C. Armando Arciniega Coron
Dra. Veridiana Reyes

Grupo de Propiedades Ópticas de la Materia (GPOM)

Centro de Investigaciones en Óptica, A.C. (CIO)

Dr. Oracio Barbosa García

Dr. Marco A. Meneses

Laboratorio de Arqueológía (LA)

Centro Universitario-Universidad Autónoma del Estado de México (CU-UAEM)

Dr. Rubén Nieto Hernández

Laboratorio de Análisis de Imágenes y Modelado Analógico (LAIMA)

Instituto de Geología-Universidad Autónoma de San Luis Potosí (Geología-UASLP)

Dr. Damiano Sarocchi 


\begin{tabular}{|c|c|c|}
\hline Laboratorio de Paleozoología (LPZOo) & Laboratorio de Análisis y Diagnóstico del Patrimonio (Ladipa) & $\begin{array}{l}\text { Grupo de Investigación de Cultura Material y Patrimo- } \\
\text { nio Cultural (GICMPC) }\end{array}$ \\
\hline $\begin{array}{l}\text { Instituto de Investigaciones Antropológicas-Universidad Nacional } \\
\text { Autónoma de México (IIA-UNAM) }\end{array}$ & El Colegio de Michoacán (Colmich) & $\begin{array}{l}\text { Facultad de Ciencias Sociales y Humanidades-Universi- } \\
\text { dad Autónoma de San Luis Potosí (FCSH-UASLP) }\end{array}$ \\
\hline Dr. Raúl Valadez Azúa & Dra. Mirta Insaurralde Caballero & Dr. Niklas Schulze \\
\hline Laboratorio Universitario de Radiocarbono (LUR) & Laboratorio de Zooarqueología (LZoo) & $\begin{array}{l}\text { Laboratorio de Análisis Químicos de Materiales en } \\
\text { Restauración (LAQMR) }\end{array}$ \\
\hline $\begin{array}{l}\text { Instituto de Geofísica, Instituto de Investigaciones Antropológicas, } \\
\text { Instituto de Geología-Universidad Nacional Autónoma de Méxi- } \\
\text { co (Geofísica, IIA, Geología-UNAM) }\end{array}$ & $\begin{array}{l}\text { Facultad de Ciencias Antropológicas-Universidad Autónoma de } \\
\text { Yucatán (FCA-UAdY) }\end{array}$ & $\begin{array}{l}\text { Facultad de Hábitat-Universidad Autónoma de San Luis } \\
\text { Potosí (FH-UASLP). }\end{array}$ \\
\hline $\begin{array}{l}\text { Dra. Laura Baramendi Orozco } \\
\text { M. en C. Galia González Hernández }\end{array}$ & Dr. Christopher Götz † & $\begin{array}{l}\text { Dra. Lilia Narváez Hernández } \\
\text { Dra. Juana María Miranda Vidales }\end{array}$ \\
\hline $\begin{array}{l}\text { Laboratorio Universitario de Caracterización Espectroscópica } \\
\text { (LUCE) }\end{array}$ & Laboratorio de Bioarqueología (LBarq) & $\begin{array}{l}\text { Laboratorio de Conservación de Materiales de Interés } \\
\text { Histórico y Artístico (LCMIHA) }\end{array}$ \\
\hline $\begin{array}{l}\text { Centro de Ciencias Aplicadas y Desarrollo Tecnológico-Universi- } \\
\text { dad Nacional Autónoma de México (CCADET-UNAM) }\end{array}$ & $\begin{array}{l}\text { Facultad de Ciencias Antropológicas-Universidad Autónoma de } \\
\text { Yucatán, (FCA-UAdY) }\end{array}$ & $\begin{array}{l}\text { Centro de Investigación en Corrosión-Universidad Autó- } \\
\text { noma de Campeche (CICORR-UACM) }\end{array}$ \\
\hline Dr. José Manuel Saniger Blesa & Dra. Vera Tiesler Blos & Dr. Javier Reyes Trujeque \\
\hline Laboratorio de Nano y Biomateriales (LANNBIO) & Taller de Análisis Químicos y Microscópicos (TAQM). & Seminario Taller de Restauración de Metales (STRM) \\
\hline $\begin{array}{l}\text { Centro de Investigación y de Estudios Avanzados-Instituto Politéc- } \\
\text { nico Nacional (CINVESTAV [Unidad Mérida]-IPN) }\end{array}$ & $\begin{array}{l}\text { Facultad de Ciencias Antropológicas-Universidad Autónoma de } \\
\text { Yucatán (FCA-UAdY) }\end{array}$ & $\begin{array}{l}\text { Escuela Nacional de Conservación Restauración y } \\
\text { Museografía-Instituto Nacional de Antropología e Histo- } \\
\text { ria (ENCRYM-INAH) }\end{array}$ \\
\hline Dra. Patricia Quintana Owen & Dra. Lilia Fernández Souza & Mtra. Jannen Contreras Vargas \\
\hline Cuerpo Académico Consolidado (CAC) & Laboratorio de Cerámica (LC) & \\
\hline $\begin{array}{l}\text { Facultad de Ingeniería Civil-Universidad Michoacana San Nicolás } \\
\text { de Hidalgo (FIC-UMSCH) }\end{array}$ & $\begin{array}{l}\text { Facultad de Ciencias Antropológicas-Universidad Autónoma de } \\
\text { Yucatán (FCA-UAdY) }\end{array}$ & $\begin{array}{l}\text { DE CIENCIAS APLICADAS A LA } \\
\text { INVESTIGACIÓN Y CONSERVACIÓN } \\
\text { DEL PATRIMONIO CULTURAL }\end{array}$ \\
\hline Dra. Elia Mercedes Alonso Guzmán & Mtra. Socorro del Pilar Jiménez Álvarez & \\
\hline
\end{tabular}

FIGURA 5. Laboratorios, grupos de investigación e instituciones participantes en la Red de Ciencias Aplicadas a la Investigación y la Conservación del Patrimonio Cultural (Red-CAICPC) (Cortesía: Red-CAICPC).

rrollo nacional, incrementar el impacto regional de los grupos de investigación mediante acciones de colaboración estratégica que implican, por ejemplo, la utilización compartida de equipos, el intercambio de experiencias y la formación especializada. En efecto, uno de sus aportes más significativos ha sido fortalecer los vínculos habidos entre los laboratorios y diversos grupos de investigación, así como ocasionar la creación de diferentes plataformas de colaboración, con lo que se han formulado y compartido protocolos comunes, detectado otros espacios de oportunidad y líneas de trabajo, e identificado más grupos de investigación que gradualmente se integrarán a las iniciativas de la red.

\section{A manera de conclusión}

Es indudable que en un relativo corto periodo de tiempo, las diversas estrategias emprendidas por la Red-CAICPC, aparte de que han acelerado la reticulación, la articula-

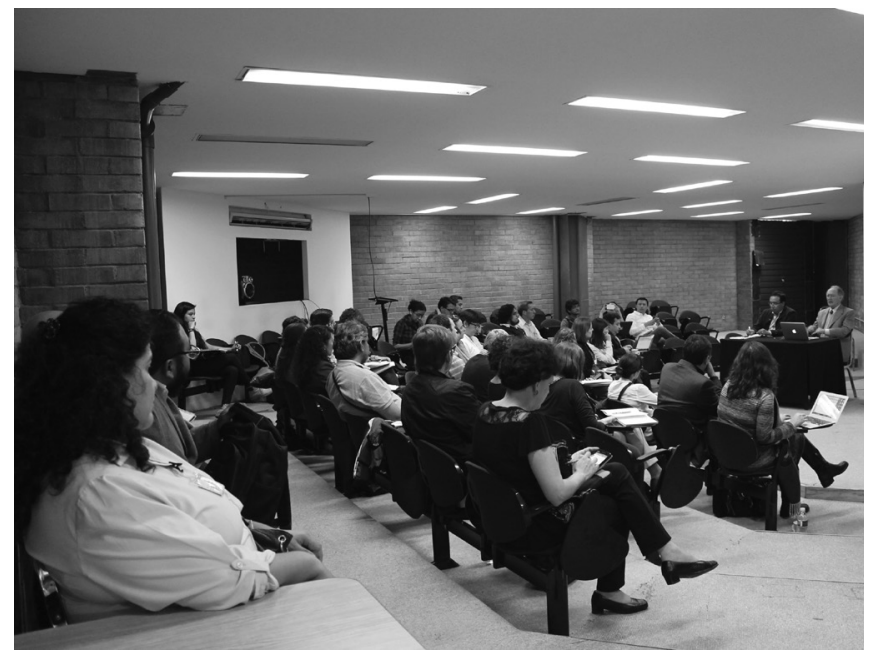

FIGURA 6. Reunión Nacional de la Red-CAICPC en la Escuela Nacional de Conservación, Restauración y Museografía (ENCRYM), del INAH, en noviembre del 2015 (Cortesía: Red-CAICPC). 
ciado las posibilidades del trabajo de investigación en el ámbito patrimonial, de creciente demanda, acelerada consolidación y gran interés en la actualidad social de nuestro país. Se espera para un futuro próximo que se amplíen sus alcances por medio de la movilidad y el intercambio de expertos, así como que se dé una mayor difusión a las investigaciones que se realizan sobre el patrimonio cultural tanto en México como en el extranjero. En alcance, la siguiente etapa contempla la creación de bases de información compartida que permitan catalizar el crecimiento de la investigación sobre patrimonio cultural en México.

Información adicional sobre la red puede obtenerse en su portal digital: [http://www.fisica.unam.mx/externos/ RedCAICPC].

\section{Referencias}

Aguilar-Téllez, Dulce María, José Luis Ruvalcaba-Sil, Pieterjan Claes y Diana González-González

2014 "False Color and Infrared Imaging for the Identification of Pigments in Paintings", Materials Research Society Symposium Proceedings, 1618:3-16, documento electrónico disponible en [http://journals.cambridge.org/action/dis playAbstract? fromPage $=$ online $\&$ aid $=9269539]$, consultado en mayo del 2016.

Alcántara García, Jocelyn, José Luis Ruvalcaba-Sil y Marie Vander Meeren

2014 "XRF Study of Mexican Iron Gall Inks: Historical and Geographical Overview of their Chemistry", Materials Research Society Symposium Proceedings, 1618:31-44, documento electrónico disponible en [http://journals.cambridge.org/action/displayAbstract?fromPage $=$ online \&a id=9268374], consultado en mayo del 2016.

Alonso, Alejandra, Nora Pérez, José Luis Ruvalcaba-Sil, Edgar Casanova, Pieterjan Claes, V. Aguilar Melo y J. Cañetas

2014 "Comparative Spectroscopic Analysis of Maya wall Paintings from Ek'Balam, Mexico", Materials Research Society Symposium Proceedings, 1618:63-72, documento electrónico disponible en [http://journals.cambridge.org/ action/displayAbstract?fromPage $=$ online $\&$ aid $=9268380]$, consultado en mayo del 2016.

Arano, Diana E., Yolanda Espinosa, Helena Barba, José Luis Ruvalcaba-Sil y Jorge A. González-Sánchez

2014 "Characterization and Evaluation of Salty Cleaning Compounds Study in Historic Objects Made of Copper Alloys from the El Tejas shipwreck", Materials Research Society Symposium Proceedings, 1618:247-256, documento electrónico disponible en [http://journals.cambridge.org/ action/displayAbstract?fromPage $=$ online $\&$ aid $=9269359]$, consultado en mayo del 2016.

Barba Pingarrón, Luis

2000 "Localización de asentamientos prehispánicos bajo el pavimento del sur de la Ciudad de México", Antropológicas, 17:59-66.
2013 Proyecto de la Red Temática de Ciencias Aplicadas a la Investigación y Conservación del Patrimonio Cultural (Red-CAICPC), documento inédito, México, Conacyt.

Cabrero, María Teresa y José Luis Ruvalcaba-Sil

2013 "Cascabeles de cobre en la cultura Bolaños, Jalisco", Arqueología Iberoamericana, 19:24-36, documento electrónico disponible en [http://www.laiesken.net/arqueologia/pdf/2013/Al1902.pdf], consultado en mayo del 2016.

Castillo Acal, David y Mario Zimmermann

2014 "Propuesta para la reconstrucción de rituales mayas prehispánicos por medio de la zooarqueología y paleobotánica", Christopher Götz, Javier Rivas, Miguel Cárdenas, Héctor Hernández, Mario Zimmermann y Carolina Ramos (coords.), Memorias del Congreso Internacional de Culturas Americanas y su Ambiente: Perspectivas desde la Zooarqueología, Paleoetnobotánica y Etnobiología, Mérida, UAdY, 204-210.

Chi Keb, J. R., V. M. Albertos González, A. Ortega y V. G. Tiesler 2013 "A New Reference Collection of Documented Human skeletons from Mérida, Yucatán, México", HOMO Journal of Comparative Human Biology, 64(5):366-376, documento electrónico disponible en [http://www.ncbi.nlm.nih. gov/pubmed/23830157], consultado en mayo del 2016.

Cucina, Andrea (ed.)

2013 Dinámicas poblacionales y afinidades biológicas entre los antiguos maya. Una perspectiva multidisciplinaria, México, UAdY.

2015 Archaeology and Bioarchaeology of Population Movement Among the Prehispanic Maya, Nueva York, Springer.

Cucina, Andrea y Allan Ortega Muñoz

2014 "Afinidades biológicas de la población de Oxtankah en el ámbito regional de la península de Yucatán durante el periodo Clásico", Estudios de Cultura Maya, 44:59-84, documento electrónico disponible en [https://revistas-filologicas.unam.mx/estudios-cultura-maya/index.php/ecm/article/view/119/119], consultado en mayo del 2016.

Cucina, Andrea y V. G. Tiesler

2014 "Mortuary Pathways and Ritual Meanings Related to Maya Human Bone Deposits in Subterranean Contexts", en Gabriel D. Wrobel (ed.), The Bioarchaeology of Space and Plac: Ideology, Power, and Meaning in Maya Mourtuary Contexts, Nueva York, Springer, 225-254.

Espinosa-Morales, Yolanda, Javier Reyes-Trujeque, Diana AranoRecio, Rosario Domínguez-Carrasco, José Luis Ruvalcaba-Sil y Patricia Quintana Owen

2014 "Deterioration of Pre-hispanic stucco masks from the Archaeological Mayan Site of Edzná, Campeche, México", Materials Research Society Symposium Proceedings, 1618:207-216, documento electrónico disponible en [http://journals.cambridge.org/action/displayAbstract?f romPage $=$ online $\& a i d=9268389]$, consultado en mayo del 2016.

Espinosa Pesqueira, Manuel E. y Elsa Arroyo Lemus 2014 "The Use of Smalt in Simon Pereyns Panel Paintings: Intentional Use and Color Changes", Materials Research Society Symposium Proceedings, 1618:131-139, documento 
electrónico disponible en [http://journals.cambridge.org/ action/displayAbstract?fromPage=online \&aid=9269353], consultado en mayo del 2016.

Fernández Souza, Lilia, Lourdes Toscano y Mario Zimmermann 2014 "De maíz y de cacao: aproximaciones a la cocina de las élites mayas en tiempos prehispánicos", en Igor Ayora Díaz y Gabriela Vargas Cetina (eds.), Estética y poder en la ciencia y la tecnología, Mérida, UAdY, 107-130.

Filloy Nadal, Laura

1992 "La conservación de la madera arqueológica en contextos lacustres: la Cuenca de México", tesis de licenciatura en restauración de bienes muebles, México, Escuela Nacional de Conservación, Restauración y Museografia, ENCRYM-INAH.

Filloy Nadal, Laura, Diana Magaloni Kerpel, José Luis Ruvalcaba-Sil y R. Sánchez Hernández

2013 "Las materias primas utilizadas para la manufactura de las figurillas y las hachas de la Ofrenda 4 de La Venta: caracterización y fuentes de origen", en Diana Magaloni Kerpel y Laura Filloy Nadal, La Ofrenda 4 de La Venta. Un tesoro olmeca reunido en el Museo Nacional de Antropología. Estudios y catálogo razonado, México, INAH, 103-127.

García-Bucio, María Angélica, Edgar Casanova-González y José

Luis Ruvalcaba-Sil

2014 "Raman Spectroscopy for the Study of XVI-XVII Centuries Colonial Paintings", Materials Research Society Symposium Proceedings, 1618:141-152, documento electrónico disponible en [http://journals.cambridge.org/action/display Abstract?fromPage $=$ online $\&$ aid $=9269526]$, consultado en mayo del 2016.

García-Hernández, M., A. García-Murillo, F. de J. Carrillo-Romo, Á. de J. Morales-Ramírez, M. A. Meneses-Nava, B. González-Penguelly y V. Garibay Febles

2013 "Effect of Starting Materials on the Morphological and Optical Properties of Er Doped $\mathrm{BaTiO}_{3}$ NanocrystaIline Films", Materials Transactions, 54 (5):806-810, documento electrónico disponible en [http://ci.nii.ac.jp/ naid/10031168429], consultado en mayo del 2016.

Götz, Christopher M.

2013 "La fauna vertebrada arqueológica de la costa campechana: el caso de Champotón y Uaymil", en Rafael Cobos (coord.), Arqueología de la costa de Campeche: la época prehispánica, Mérida, UADY, 97-123.

2013 "Introduction", en Christopher M. Götz y Kitty F. Emery (eds.), The Archaeology of Mesoamerican Animals, Atlanta, Lockwood Press, 1-22.

2014 "The Sustainability of Agroecosystems of the Prehispanic Maya-implications of Hunting and Animal Domestication in the Northern Maya lowlands", en Travis W. Stanton (ed.), The Archaeology of Yucatan: New Directions and Data, Oxford, British Archeological Reports (BAR), 477-486.

Götz, Christopher M. y Kitty F. Emery (eds.)

2014 La arqueología de los animales de Mesoamérica, Atlanta, Lockwood Press.

Götz, Christopher M. y Travis W. Stanton

2013 "The Use of Animals by the Prehispanic Maya of the Northern lowlands", en Christopher M. Götz y Kitty F. Emery (eds.), The Archaeology of Mesoamerican Animals, Atlanta, Lockwood Press, 191-232.

Götz, Christopher M., Raúl Valadez Azúa y Bernardo Rodríguez Galicia

2014 "The Archaeology of the Interaction between Marine Mammals and Humans in Central and Southeastern Mesoamerica", en S. Muñoz, C. Götz y E. Ramos (eds.), Neotropical and Caribbean Aquatic Mammals: Perspectives from Archaeology and Conservation Biology, 25-58, Nueva York, Nova Science Publishers.

Herrera Flores, David A. y Christopher M. Götz 2014 "La alimentación de los antiguos mayas de la Península de Yucatán: consideraciones sobre la identidad y la cuisine en la época prehispánica", Estudios de Cultura Maya, XLIII: 69-98, documento electrónico disponible en [http://www.redalyc.org/articulo.oa?id=281331367003], consultado en mayo del 2016.

Jiménez, Leticia, Diana E. Arano, José Luis Ruvalcaba-Sil y Fanny Unikel

2014 "Characterization of inherent materials of san Antonio Altarpiece in San Roque church, Campeche", Materials Research Society Symposium Proceedings, 1618:153160, documento electrónico disponible en [http://journals. cambridge.org/action/displayAbstract?fromPage=online\&a id=9272511], consultado en mayo del 2016.

Magaloni, Diana

1990 "Metodología para el análisis de la técnica pictórica mural prehispánica: el Templo Rojo, Cacaxtla", tesis de licenciatura en restauración de bienes muebles, México, ENCRYM-INAH.

Manrique-Ortega, M. D., P. Claes, Edgar Casanova-González, José Luis Ruvalcaba-Sil, María Aangélica García-Bucio y L. Lowe

2014 "Non-invasive Analysis of Green stone Pieces from Tomb 1 of Chiapa de Corzo, Chiapas", Materials Research Society Symposium Proceedings, 1618:17-30, documento electrónico disponible en [http://journals.cambridge.org/ action/displayAbstract?fromPage=online\&aid=9269341], consultado en mayo del 2016.

McGlinchey, Ch., A. Aviram, Sandra Zetina Ocaña, Elsa Arroyo Lemus, Renato González Mello, José Luis Ruvalcaba-Sil y Manuel Espinosa Pesqueira

2013 "David Alfaro Siqueiros: Revolutionary Discoveries from a can of Paint", Journal of the American Institute for Conservation, 52 (4):278-289.

Medina-González, Isabel 1998 "Jícaras prehispánicas policromadas procedentes de contextos arqueológicos húmedos. Análisis integral de un artefacto arqueológico", tesis de licenciatura en restauración de bienes muebles, México, ENCRYM-INAH.

Melgar Tisoc, Emiliano R. y José Luis Ruvalcaba-Sil 2014 "Technological Analysis of the Calcite Beads from the Great Temple of Tenochtitlan", Materials Research Society Symposium Proceedings, 1618:97-108, documento electrónico disponible en [http://journals.cambridge.org/ac- 
tion/displayAbstract?fromPage =online $\&$ aid $=9269330 \&$ filel $\mathrm{d}=$ S194642741400459X], consultado en mayo del 2016.

Muñoz, Sebastián, Christopher M. Götz y Elizabeth Ramos Roca (eds.)

2014 Neotropical and Caribbean Aquatic mammals: Perspectives from Archaeology and Conservation Biology, Hauppauge, Nueva York, Nova Science Publishers.

Ortega, A. y A. Tiesler

2013 "La antropología física y la bioarqueología: reflexiones de su lugar institucional en el estudio de las poblaciones alguna vez vivas en el ámbito nacional", Estudios de Antropología Biológica 15:399-413, documento electrónico disponible en [https://www.academia. edu/1175959/Ortega_Allan_y_Vera_Tiesler_2011_._ La_antropolog\%C3\%ADa_f\%C3\%ADsica_y_la_ bioarqueolog\%C3\%ADa_reflexiones_de_su_lugar_institucional_en_el_estudio_de_las_poblaciones_alguna_vez_vivas_en_el_\%C3\%A1mbito_nacional], consultado en mayo del 2016.

2014 "New Isotope Data on Maya Mobility and Enclaves at Classic Copan, Honduras", Journal of Anthropological Archaeology, 36:32-47, documento electrónico disponible en [http://www.sciencedirect.com/science/article/pii/ S0278416514000154], consultado en mayo del 2016.

Perea, A., P. C. Gutiérrez; A. Climent-Font, S. Rovira-Llorens, P. Fernández-Esquivel, José Luis Ruvalcaba-Sil, A. Verde y Alessandro Zucchiatti

2013 "Prehispanic goldwork technology. The Quimbaya Treasure, Colombia", Journal of Archaeological Science, 40 (5):2326-2334, documento electrónico disponible en [http://www.sciencedirect.com/science/article/pii/ S0305440313000022], consultado en mayo del 2016.

Price, T. D., J. H. Burton, P. D. Fullagar, L. E. Wright, J. E. Buikstra y $\mathrm{V}$. Tiesler

2013 "Los isótopos de estroncio en el estudio de la movilidad entre los antiguos mayas", en A. Cucina (ed.), Afinidades biológicas y dinámicas poblacionales entre los antiguos mayas. Una visión multidisciplinaria, México, UAdY, 111-128.

Quintana, Patricia, V. Tiesler, M. Conde, R. Trejo, C. Bolio, J. L. Alvarado y D. Aguilar

2014 "Spectrochemical Characterization of Red Pigments used in Classic Period Maya Funerary Practices", Archaeometry, 57 (6):1045-1059, documento electrónico disponible en [http://onlinelibrary.wiley.com/doi/10.1111/ arcm.12144/abstract], consultado en mayo del 2016.

Ramírez-Salomón, M., E. Vega-Lizama, V. Tiesler, G. AlvaradoCárdenas, M. López-Villanueva, T. Sierra Sosa y A. Cucina

2014 "The C-shaped Canal Molar: an Endodontic-Archaeological Study of the Relationships between Mayan Pre-Hispanic and Contemporary Population of Yucatan", International Endodontic Journal, 47 (11): 1084-1089, documento electrónico disponible en [http://onlinelibrary. wiley.com/doi/10.1111/iej.12255/abstract], consultado en mayo del 2016.

Red-CAICPC

2016 Red de Ciencias Aplicadas a la Investigación y Con- servación del Patrimonio Cultural (Red-CAICPC) [Página web], documento electrónico disponible en [http://www. fisica.unam.mx/externos/RedCAICPCl, consultado en marzo del 2016.

Riquelme, Francisco, J. Alvarado-Ortega, José Luis RuvalcabaSil, M. Aguilar-Franco y H. Porras-Múzquiz

2013 "Chemical Fingerprints and Microbial Biomineralization of fish Muscle Tissues from the late Cretaceous Múzquiz Lagerstätte, México", Revista Mexicana de Ciencias Geológicas, 30 (2):417-435, documento electrónico disponible en [http://scielo.unam.mx/pdf/rmcg/v30n2/ v30n2a12.pdf], consultado en mayo del 2016.

Riquelme, Francisco, P. Northrup, José Luis Ruvalcaba-Sil, V. Stojanoff, D. P. Siddons y J. Alvarado-Ortega

2014a "Insights into Restricted Organic Preservation in Chiapas amber Using IR Microimaging, PIXE/RBS and Sulfur K-edge XANES spectroscopy", Journal of Applied Physics A, 116:97-109.

Riquelme, Francisco, José Luis Ruvalcaba-Sil, Jesús AlvaradoOrtega, Emilio Estrada-Ruiz, Martin Galicia-Chávez, Héctor Porras-Múzquiz, Vivian Stojanoff, D. Peter Siddons y Lisa Miller

2014b "Amber from México: Coahuilite, Simojovelite and Bacalite", Materials Research Society Symposium Proceedings, 1618:169-180, documento electrónico disponible en [http://journals.cambridge.org/action/displayAbstract?fromP age=online\&aid=9269555], consultado en mayo del 2016.

Ruvalcaba-Sil, José Luis, Edgar Casanova González, Nora Ariadna Pérez-Castellanos y María Angélica García-Bucio

2013 "HERAS: A helium jet to Prevent Damage on Works of art in Raman Experiments", Journal of Raman Spectroscopy, 44 (12):1711-1717, documento electrónico disponible en [http://onlinelibrary.wiley.com/doi/10.1002/jrs.4402/ abstract], consultado en mayo del 2016.

Sánchez Martínez, Manuel y Dolores Elena Álvarez Gasca 2013 "Nanopartículas de $\mathrm{Ca}(\mathrm{OH})$ en la conservación del mortero de cal-arena de la Noria Alta de Cata en Guanajuato, Gto.", ponencia presentada en el 48 Congreso Mexicano de Química, 31 de agosto al 4 de septiembre, Guanajuato, México, Universidad de Guanajuato.

2014 "Los agentes que deterioran los materiales de los objetos del Patrimonio Cultural", ponencia presentada en el III Foro de Conservación y Restauración del Patrimonio Cultural, 26 de septiembre, México, Universidad Autónoma de Querétaro.

Sierra Sosa, T., A. Cucina, T. Douglas Price, J. H. Burton y V. Tiesler 2014 "Maya Coastal Production, Exchange, Life Style, and Population Mobility: a View from the Classic Period port of Xcambó, Yucatán, México", Ancient Mesoamerica, 25(01):221-238, documento electrónico disponible en [https://www.researchgate.net/publication/271897387_ Maya_coastal_production_exchange_and_population_ mobility_A_view_from_the_Classic_period_port_of_ Xcambo_Yucatan_Mexico], consultado en mayo del 2016.

Solís Ciriaco, Reyna B. y José Luis Ruvalcaba-Sil 2014 "Provenance and Manufacture of Mixtec Style Objects found on the Surrounding Structures of the Precinct of 
the Great Temple of Tenochtitlan", Materials Research Society Symposium Proceedings, 1618:121-130, documento electrónico disponible en [http://journals.cambridge.org/ action/displayAbstract?fromPage=online \&aid=9269507], consultado en mayo del 2016.

Tiesler, V. G.

2014 The Bioarchaeology of Artificial Cranial Modifications. New Approaches to Head Shaping and its Meanings in Pre-Columbian Mesoamerica and Beyond, Nueva York, Springer.

Tiesler, V. G., A. Coppa, P. Zabala y A. Cucina

2014 "Scurvy-related morbidity and death among Christopher Columbus' crew at La Isabela, the first European town in the New World (1494-1498)", International Journal of Osteoarchaeology, 26 (2):191-202, documento electrónico disponible en [http://onlinelibrary.wiley.com/doi/10.1002/ oa.2406/abstract], consultado en mayo del 2016.

Tiesler, V. G. y A. Cucina

2014 "Past, Present and Future Perspectives in Maya Bioarchaeology: a view from Yucatan, Mexico", en O’Donnabhain Barra y María Cecilia Lozada Cerna (eds.), Archaeological Human Remains. Global Perspectives, Nueva York, Springer, 165-176.

Tiesler, V. G., A. Cucina, Patricia Quintana, D. Aguilar, I. Oliva, J. V. Cauich, W. J. Rodríguez Folan y M. R. Domínguez

2013a "What's on the bone? Interdisciplinary approaches in Reconstructing the Posthumous Body Treatment of the Ancient Maya Aristocracy of Calakmul, Campeche, Mexi-

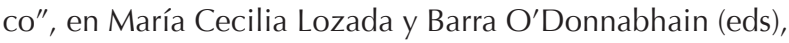
The Dead Tell Tales. Essays in Honor of Jane E. Buikstra, Los Ángeles, Cotsen Institute-UCLA, 85-94.

Tiesler, V. G., A. Romano, J. Gómez y A. Daneels 2013b "Posthumous Body Manipulation in the Classic Period Mixtequilla. Re-evaluating the Human Remains of Ossuary I from El Zapotal, Veracruz", Latin American Antiquity, 24 (1):47-71, documento electrónico disponible en [https://www.jstor.org/stable/43746259?seq=1\#page_ scan_tab_contents], consultado en mayo del 2016.

Velázquez-Castro, Adrián

2014 "Huastec shell Objects in the Templo Mayor Offerings of Tenochtitlan", Materials Research Society Sym- posium Proccedings, 16118: 89-96, documento electrónico disponible en [http://journals.cambridge.org/action/ displayAbstract? fromPage $=$ online $\&$ aid $=9268386 \&$ fileld =S1946427414004588], consultado en mayo del 2016.

Velázquez-Castro, Adrián y Arnd Adje Both

2014 "El sonido de la tierra. Cascabeles de Oliva mexicas", en Francisca Zalaquett R., Martha Ilia Nájera C. y Laura Elena Sotelo S. (eds.), Entramados sonoros de tradición mesoamericana. Identidad imágenes y contextos, México, UNAM, 17-50.

Velázquez-Castro Adrián y Emiliano Melgar Tísoc 2014 "Producciones palaciegas tenochcas en objetos de concha y lapidaria", Ancient Masoamerica, 25 (2014): 295308, documento electrónico disponible en [http://journals. cambridge.org/action/displayAbstract?fromPage=online\&a $\mathrm{id}=9315386]$, consultado en mayo del 2016.

Villegas, Pascale, Antonio Rodríguez, Manuel Espinosa Pesqueira, Ma. del Rosario Domínguez y Javier Reyes Trujeque

2014 "Caracterización mineralógica y petrográfica de las tejas de barro utilizadas en Campeche en diferentes épocas", en Pascale Villegas (ed.), Del puerto de Marsella a las casas de Campeche-El comercio de las tejas de barro (1852 a 1932), México, UAC, 103-115.

Zetina Ocaña, Sandra, José Luis Ruvalcaba-Sil, Tatiana Falcón, J. Arenas Alatorre, S. Yanagisawa, M. Álvarez Icaza Longoria y Eumelia Hernández

2014 "Material Study of the Codex Colombino", en A. Sgamelloti, B. Brunetti, C. Miliani (eds.), Science and Art: The Painted Surface, Cambridge, Royal Society of Chemistry, 120-146.

Zucchiatti, Alessandro, A. Climent Font, P. C. Gutiérrez Neira, A. Perea, P. Fernández Esquivel, S. Rovira LLorens, José Luis Ruvalcaba-Sil y A. Verde

2014 “Prehispanic GoldworkTechnology Study by PIXEAnalysis", Nuclear Instruments and Methods B, 332:160-164, documento electrónico disponible en [http://www.sciencedirect.com/science/article/pii/S0168583X14003097], consultado en mayo del 2016. 


\section{Luis Barba Pingarrón}

Instituto de Investigaciones Antropológicas (IIA),

Universidad Nacional Autónoma de México, (UNAM), México

barba@unam.mx

Ingeniero químico industrial (Instituto Politécnico Nacional [IPN], México), maestro en geología arqueológica (University of Georgia [UGA], Estados Unidos de América [EUA]) y doctor en antropología (Universidad Nacional Autónoma de México [UNAM], México). Desde hace 40 años es académico del Instituto de Investigaciones Antropológicas (IIA-UNAM, México); fue fundador y es el actual coordinador del Laboratorio de Prospección Arqueológica (LPA) de dicho instituto. Ha impartido cursos en multitud de instituciones educativas nacionales y también extranjeras, como en el Perú, España e Italia. Es miembro del Sistema Nacional de Investigadores (SNI), nivel III (Conacyt), de los programas de estímulos de la UNAM, nivel D, y de la Academia Mexicana de Ciencias (AMC). Cuenta con más de 120 artículos en publicaciones nacionales e internacionales, así como con varios libros y capítulos de libros. Sus principales líneas de investigación se inscriben en el campo de la arqueometría. También es pionero en el campo de estudio de los residuos químicos en pisos arqueológicos, así como en el de residuos químicos en recipientes cerámicos en México. Recientemente ha trabajado en el análisis de los materiales constructivos de estructuras arquitectónicas prehispánicas. Es el responsable de la Red de Ciencias Aplicadas a la Investigación y Conservación del Patrimonio Cultural (Red-CAICPC), Conacyt.

\section{Isabel Medina-González}

Escuela Nacional de Conservación, Restauración y Museografía (ENCRyM), Instituto Nacional de Antropología e Historia (INAH), México

isabel_medina_g@encrym.edu.mx

Licenciada en restauración de bienes muebles (Escuela Nacional de Conservación, Restauración y Museografía [ENCRym], Instituto Nacional de Antropología e Historia [INAH], México); maestra en gestión de patrimonio arqueológico (University of York, Reino Unido) y doctora en arqueología (University College London [UCL], Reino Unido). Desde 1993 ha trabajado en el INAH como restauradora, investigadora y docente. En el año 2000 fue galardonada con el Premio Internacional al Joven Americanista, del Congreso Internacional de Americanistas. Ha sido miembro honorario del Institute of Archaeology (UCL, Reino Unido). Actualmente es profesora de tiempo completo en la licenciatura y el posgrado de la ENCRYM-INAH, así como miembro del Sistema Nacional de Investigadores (SNI), nivel I, del Consejo Nacional de Ciencia y Tecnologia (Conacyt, México), y del Comité del Laboratorio de Diagnóstico de Obras de Arte (LDOA), Instituto de Investigaciones Estéticas (IIE), Universidad Autónoma de México (UNAM); también es secretaria académica de ıCOMOS-México (todos en México).

Postulado/Submitted 25.05.16

Aceptado/Accepted 15.06.16

Publicado/Published 11.07.16 